\title{
Estimativa da variação da produtividade do arroz em diferentes escalas temporais do índice SPI
}

\author{
Diego Simões Fernandes ${ }^{(1)}$ e Alexandre Bryan Heinemann ${ }^{(1)}$ \\ (1)Embrapa Arroz e Feijão, Rodovia GO 462, Km 12, Zona Rural, CEP 75375-000 Santo Antônio de Goiás, GO.E-mail: diegosifer@cnpaf.embrapa.br, \\ alexbh@cnpaf.embrapa.br
}

Resumo - O objetivo deste trabalho foi avaliar a acurácia do índice de seca SPI (índice padronizado de precipitação), em diferentes escalas temporais, na determinação da ocorrência de eventos de seca e na estimativa de seus efeitos na produtividade do arroz de terras altas, em seis diferentes microrregiões do Estado de Goiás. Foram utilizados dados diários de precipitação, provenientes de estações pluviométricas localizadas nos municípios de Uruaçu, Aruanã, Alexânia, Mineiros, Catalão e Itumbiara, no Estado de Goiás, como também a série histórica de produtividade do arroz de terras altas para as microrregiões goianas de Porangatu, Rio Vermelho, Entorno de Brasília, Sudoeste de Goiás, Catalão e Meia-Ponte. As escalas temporais utilizadas foram: mensal, trimestral, semestral e anual. As diferenças entre as escalas temporais do SPI foram avaliadas quanto ao número e frequência dos eventos de seca, ao percentual de acerto na estimativa da variação da produtividade ajustada de arroz, e quanto aos desvios entre essas estimativas e a produtividade observada. O aumento na escala temporal do SPI possibilita a identificação de eventos de seca de maior duração, e a escala anual (SPI-12) apresentou o melhor desempenho na estimativa da variação da produtividade ajustada de arroz de terras altas.

Termos para indexação: arroz de terras altas, índice de seca, produtividade.

\section{Rice yield variability estimates at different time scales of SPI index}

\begin{abstract}
The objective of this work was to evaluate the accuracy of the drought index SPI (standardized precipitation index), at different time scales, for determining the drought occurrences and for estimate their effects on upland rice adjusted yield in six different microregions in Goiás state, Brazil. Precipitation daily data were used from rain gauge stations located at Uruaçu, Aruanã, Alexânia, Mineiros, Catalão and Itumbiara, in Goiás state, as well as upland rice yield data set for the microregions Porangatu, Rio Vermelho, Entorno de Brasília, Southwest Goiás, Catalão and Meia-Ponte, also located in Goiás state. The temporal time scales used in this study were monthly, quarterly, semiannual and annual. The differences among the SPI temporal scales were evaluated for the number and frequency of drought occurrences, the percentage accuracy of the variation estimate related to the upland rice adjusted yield data, and the deviations between these estimates and the observed yield. Increasing SPI temporal scale makes it possible the identification of persistent drought, and the annual scale (SPI-12) showed the best performance for estimating the variability of upland rice adjusted yield.
\end{abstract}

Index terms: upland rice, drought index, yield.

\section{Introdução}

A seca é considerada um fenômeno climático extremo que tem-se tornado uma ameaça global (Burke et al., 2005). Eventos de seca podem ter impacto significativo nos diversos setores da atividade humana. Altas temperaturas, ventos fortes, baixa umidade do ar, baixa capacidade de retenção de água no solo e o aumento no potencial erosivo das chuvas contribuem para intensificar a gravidade das secas (Freitas, 2005; Salas et al., 2005). Esse fenômeno pode ser classificado em quatro tipos: meteorológico, agrícola, hidrológico e socioeconômico (Heim, 2002).
O arroz de terras altas é considerado altamente sensível à deficiência hídrica (Pinheiro, 2006). Em razão disso, há necessidade de se registrar a intensidade e a ocorrência desses eventos, por meio de ferramentas como os índices de seca. Isso possibilita a caracterização ambiental de acordo com a probabilidade de ocorrência de eventos extremos, o que auxilia os programas de melhoramento vegetal na indicação de genótipos mais adequados aos ambientes. Entre os índices de seca, o índice padronizado de precipitação (SPI) foi desenvolvido com a finalidade de quantificar deficits e excessos de precipitação, em uma determinada região, em relação à precipitação normal (McKee et al., 1993). 
Esse índice é geralmente utilizado para identificar "secas meteorológicas", e destaca-se por ser obtido por meio de apenas uma variável climática, a precipitação. Além disso, o SPI não é afetado pela topografia e permite a comparação quantitativa da ocorrência de seca em diferentes regiões e em múltiplas escalas temporais, pelo fato de ser normalizado (Michaelides \& Pashiardis, 2008; Fathabadi et al., 2009). Vários estudos têm mostrado que o SPI é útil para caracterizar ocorrência, frequência e extensão espacial e temporal das secas (Lloyd-Hughes $\&$ Saunders, 2002; Potop et al., 2010).

A aplicação desse índice na caracterização da seca está relacionada à sua capacidade de indicar a ocorrência de deficit no acúmulo de água no solo. Pelo fato de a resposta dos sistemas hidrológicos à precipitação variar em função do tempo, a escala temporal na qual ocorre a deficiência é extremamente importante (Vicente-Serrano et al., 2010). Assim, os valores do SPI, obtidos nas escalas mensal e trimestral, podem representar os efeitos da seca na agricultura, enquanto os valores de SPI, para as escalas semestral e anual, geralmente, representam os efeitos da seca nos níveis dos reservatórios de água e descarga dos rios (Keskin \& Sorman, 2010).

Esse índice já foi aplicado para o Estado de Goiás e apresentou bom desempenho na quantificação de eventos de seca (Fernandes et al., 2010). Entretanto, os autores utilizaram somente a escala mensal e aplicaramna a apenas uma localidade do Estado. Assim, é importante avaliar as diferentes escalas temporais do SPI na caracterização de eventos extremos de seca e umidade e na estimativa da variação da produtividade do arroz de terras altas, em diferentes localidades do Estado de Goiás.

O objetivo deste trabalho foi avaliar a acurácia do índice de seca SPI, em diferentes escalas temporais, na determinação da ocorrência de eventos de seca e na estimativa de seus efeitos na produtividade do arroz de terras altas, em seis diferentes microrregiões do Estado de Goiás.

\section{Material e Métodos}

A região de estudo compreende seis microrregiões do Estado de Goiás (Tabela 1 e Figura 1). Segundo Nimer (1989), essa região é caracterizada pela transição entre os climas quentes de latitudes baixas e os climas mesotérmicos do tipo temperado, de latitudes médias, e apresenta duas estações bem definidas, seca (abrilsetembro) e chuvosa (outubro-março), com índices médios de pluviosidade entre 1.000 e $1.500 \mathrm{~mm}$ (Keller Filho et al., 2005).

No presente trabalho, foram utilizadas séries históricas de precipitação diária, fornecidas por estações pluviométricas que integram a base de dados da Agencia Nacional das Águas (Brasil, 2011). A localização geográfica das estações pluviométricas, o município e microrregiões aos quais pertencem, bem como o período de dados analisados estão descritos na Tabela 1. Realizou-se uma análise de consistência com base na série histórica de dados de precipitação utilizada, tendo-se seguido a metodologia descrita por Heinemann et al. (2007).

Para o cálculo do SPI, utilizou-se a distribuição gama como distribuição de frequência da

Tabela 1. Localização geográfica das microrregiões e períodos de coleta de dados de precipitação diária.

\begin{tabular}{llcccc}
\hline Microrregião & Município & Latitude $\left({ }^{\circ}\right)$ & Longitude $\left({ }^{\circ}\right)$ & Período & Observações \\
\hline Porangatu & Uruaçu & $-14,52$ & $-49,05$ & $1969-2007$ & 39 \\
Rio Vermelho & Aruanã & $-14,92$ & $-51,08$ & $1971-2006$ & 36 \\
Entorno de Brasília & Alexânia & $-16,08$ & $-48,50$ & $1968-2007$ & 40 \\
Sudoeste de Goiás & Mineiros & $-17,69$ & $-52,88$ & $1969-2007$ & 39 \\
Catalão & Catalão & $-18,10$ & $-47,69$ & $1971-2006$ & 36 \\
Meia Ponte & Itumbiara & $-18,34$ & $-49,61$ & $1972-2006$ & 35 \\
\hline
\end{tabular}

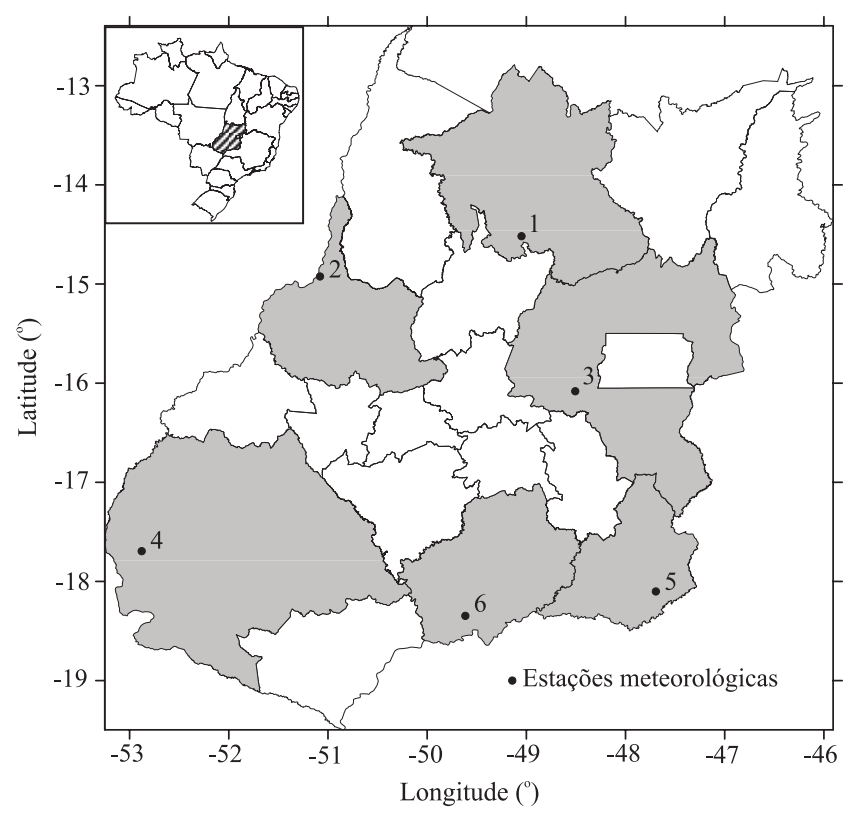

Figura 1. Distribuição espacial das microrregiões e das estações meteorológicas utilizadas neste trabalho. 
precipitação, cuja função de densidade de probabilidade é definida pela equação 1 :

$g(x)=\left(1 / \beta^{\alpha} \Gamma(\alpha)\right) x^{\alpha-1} e^{-\alpha / \beta}$, para $x>0$,

em que: $\alpha$ e $\beta$ (ambos $>0$ ) são os parâmetros que representam a forma e escala, respectivamente; $\mathrm{x}$ é a quantidade precipitada $(\mathrm{x}>0)$; e $\Gamma(\alpha)$ é a função gama, definida pela equação 2, abaixo:

$\Gamma(x)=\int_{0}^{\infty} y^{\alpha-1} e^{-y} d y$

Os parâmetros $\alpha$ e $\beta$ foram estimados com base no método de máxima verossimilhança (Thom, 1958), descrito pelas equações 3, 4 e 5:

$\alpha=1 / 4 \mathrm{~A}(1+\sqrt{1+4 \mathrm{~A} / 3})$

$\beta=\bar{x} / \alpha$

$A=\ln (\bar{x})-\sum \ln (x) / n$,

em que: n é o número de observações da precipitação.

A função de densidade de probabilidade foi integrada em relação a $\mathrm{x}$, e os parâmetros estimados $\alpha$ e $\beta$ foram utilizados para obter a equação de probabilidade cumulativa $\mathrm{G}(\mathrm{x})$, em função da precipitação ocorrida em uma determinada escala temporal, de acordo com a equação 6:

$G(x)=\int_{0}^{x} g(x) d x=1 / \beta^{\alpha} \Gamma(x) \int_{0}^{x} x^{\alpha} e^{-\alpha / \beta} d x$

Segundo $\mathrm{Wu}$ et al. (2005), substituindose $x / \beta$ por $t$, na equação 6 , ela se torna uma função gama incompleta, conforme a equação 7 :

$\mathrm{G}(\mathrm{x})=1 / \Gamma(\mathrm{x}) \int_{0}^{\mathrm{x}} \mathrm{t}^{\alpha-1} \mathrm{e}^{-\mathrm{t}} \mathrm{dt}$

Valores nulos para a precipitação $(\mathrm{x}=0)$ tornam a função gama indeterminada. Neste caso, a probabilidade cumulativa é obtida pela equação 8 :

$H(x)=q+(1-q) G(x)$,

em que: $\mathrm{H}(\mathrm{x})$ é a distribuição de probabilidade cumulativa, q é a probabilidade de ocorrência de valores nulos e $\mathrm{G}(\mathrm{x})$ é a probabilidade cumulativa teórica.
Após estas considerações, os valores do SPI podem ser obtidos pelas equações 9 e 10:

SPI $=-\left(\mathrm{t}-\mathrm{c}_{0}+\mathrm{c}_{1} \mathrm{t}+\mathrm{c}_{2} \mathrm{t}^{2} / 1+\mathrm{d}_{1} \mathrm{t}+\mathrm{d}_{2} \mathrm{t}^{2}+\mathrm{d}_{3} \mathrm{t}^{3}\right)$, para $0,0<\mathrm{H}(\mathrm{x}) \leq 0,5(9)$

SPI $=+\left(\mathrm{t}-\mathrm{c}_{0}+\mathrm{c}_{1} \mathrm{t}+\mathrm{c}_{2} \mathrm{t}^{2} / 1+\mathrm{d}_{1} \mathrm{t}+\mathrm{d}_{2} \mathrm{t}^{2}+\mathrm{d}_{3} \mathrm{t}^{3}\right)$ para $0,5<\mathrm{H}(\mathrm{x}) \leq 1,(10)$

em que os valores dos coeficientes foram: $\mathrm{c}_{0}=2,515517 ; \mathrm{c}_{1}=0,802853 ; \mathrm{c}_{2}=0,010328 ;$ $\mathrm{d}_{1}=1,432788 ; \mathrm{d}_{2}=0,189269$ e $\mathrm{d}_{3}=0,001308$; e em que $\mathrm{t}$ é definido pelas equações 11 e 12:

$\mathrm{t}=\sqrt{\ln \left[1 /(\mathrm{H}(\mathrm{x}))^{2}\right]}$, para $0<\mathrm{H}(\mathrm{x}) \leq 0,5$

$\mathrm{t}=\sqrt{\ln \left[1 /(1-\mathrm{H}(\mathrm{x}))^{2}\right]}$, para $0,5<\mathrm{H}(\mathrm{x}) \leq 1$.

No presente trabalho, o SPI foi calculado de acordo com quatro escalas temporais: SPI-1, mensal; SPI-3, trimestral; SPI-6, semestral; e SPI-12, anual. Os valores do SPI para as diferentes escalas temporais foram classificados, conforme a Tabela 2 (Giddings et al., 2005).

As séries históricas de produtividade da cultura de arroz de terras altas, provenientes do IBGE e armazenadas na base de dados Agrotec (Garagorry \& Rego, 1997), compreenderam o período de 1975 a 2006. Como esta série histórica de produtividade apresenta interação dos efeitos do avanço tecnológico e da variabilidade climática, há necessidade de minimizá-los e de ajustar os dados de produtividade para o último ano de dados disponível, ou seja, para o ano referência 2006. Para isso, uma linha de tendência foi ajustada de acordo com os dados de produtividade observados de cada microrregião, por meio do método não paramétrico de regressão não polinomial "loess"

Tabela 2. Classificação dos valores obtidos por meio do índice padronizado de precipitação (SPI).

\begin{tabular}{lc}
\hline Classe & SPI \\
\hline Extremamente úmido & $\geq 2,00$ \\
Muito úmido & 1,00 a 1,99 \\
Moderadamente úmido & 0,50 a 0,99 \\
Próximo ao normal & $-0,49$ a 0,49 \\
Moderadamente seco & $-0,50$ a $-0,99$ \\
Muito seco & $-1,00$ a $-1,99$ \\
Extremamente seco & $\leq-2,00$ \\
\hline
\end{tabular}

Fonte: adaptado de Giddings et al. (2005). 
(Cleveland, 1979). Os valores estimados representam o avanço tecnológico e os desvios relativos à variação climática (Hollinger et al., 2001). Os valores de produtividade observados foram ajustados para o ano de 2006 (último ano da base de dados Agrotec), tendose multiplicado esses valores pelos desvios. Assim, a produtividade ajustada foi obtida.

Os valores obtidos no cálculo do SPI foram analisados por meio do número de ocorrência de eventos de seca, classificados conforme a Tabela 2, em razão das diferentes escalas temporais, para as diferentes microrregiões.

Para analisar as tendências dos valores obtidos de SPI, nas diferentes escalas temporais, e a variabilidade da série histórica de produtividade ajustada de arroz de terras altas, somaram-se os valores do SPI, de cada escala temporal, tendo-se considerado somente os meses que representam o período de desenvolvimento da cultura de arroz de terras altas no Estado de Goiás, ou seja, de outubro a março. Assim, para a escala temporal mensal, os valores obtidos para os meses de outubro a março foram somados, e o índice para essa escala foi denominado de SPI-1.ac. Para a escala temporal trimestral, os valores correspondentes ao trimestre outubro, novembro e dezembro foram somados aos correspondentes ao trimestre janeiro, fevereiro e março. Neste caso, o índice foi denominado de SPI-3.ac. Para a escala semestral, utilizou-se o valor referente ao semestre composto pelos meses outubro, novembro, dezembro, janeiro, fevereiro e março, e o índice foi denominado de SPI-6.ac. Para a escala anual, não houve necessidade de soma, uma vez que, para essa escala, é calculado somente um valor por ano, denominado de SPI-12.ac. As diminuições ou aumentos dos valores da produtividade ajustada do arroz de terras altas foram classificados, tendo-se levado em conta a diferença entre o ano atual e anterior.

O percentual de acerto dos valores obtidos pelo SPI, nas diferentes escalas, em relação à estimativa da variação na produtividade ajustada de arroz de terras altas, foi obtido por meio de uma Tabela de contingência, com os seguintes critérios: acerto, mês classificado como seco, com diminuição da produtividade; erro, mês classificado como seco e com aumento da produtividade; negativo errado, mês classificado como úmido e com diminuição da produtividade; e negativo correto, mês classificado como úmido e com aumento da produtividade.
O efeito das diferentes escalas temporais do SPI, na estimativa da variação da produtividade ajustada do arroz de terras altas, também foi analisado de acordo com o desvio dessa produtividade ajustada em relação à média histórica da produtividade de cada uma das diferentes microrregiões. Esses desvios ou anomalias da produtividade ajustada foram confrontados com os valores do SPI, para as escalas temporais mensal, trimestral, semestral e anual.

\section{Resultados e Discussão}

A evolução da série temporal dos valores de SPI, nas diferentes escalas temporais, permitiu que se constatassem vários períodos secos nas seis microrregiões estudadas (Figura 2). A escala mensal (SPI-1), considerada como de alta frequência temporal, apresentou o maior número de eventos. $\mathrm{O}$ aumento na escala temporal causou uma redução na frequência temporal e diminuiu o número de eventos. Isso ocorre porque, ao se aumentar a escala temporal, o SPI comporta-se como uma média móvel que tem sua ordem (k) aumentada (Guttman, 1998; Lloyd-Hughes \& Saunders, 2002). O número de eventos extremos também é reduzido com o aumento da escala temporal (Tabela 3). Isso porque o aumento da escala temporal proporciona ao SPI a sensibilidade para identificar eventos de seca e umidade mais persistentes, ou seja, de maior duração (Livada \& Assimakopoulos, 2007). Para as escalas de alta frequência temporal (SPI-1 e SPI-3), as microrregiões que apresentaram maiores números de eventos extremos de seca foram Entorno de Brasília, Catalão, Sudoeste de Goiás e Porangatu. No entanto, para o SPI-12, de baixa frequência temporal, apenas as microrregiões Entorno de Brasília, Meia-Ponte e Porangatu apresentaram os maiores números de ocorrência de eventos extremamente secos. Assim, observou-se que a escala temporal no SPI está relacionada à persistência dos eventos de seca e umidade, ou seja, escalas com baixa frequência temporal são mais eficientes em detectar eventos extremos com maior duração e, consequentemente, com maior impacto na economia.

Em razão de este índice apresentar distribuição normal, era esperado que o maior número de ocorrência de eventos secos e úmidos se concentrasse entre os valores $-0,49$ e 0,49 , próximos ao normal (Tabela 2). 
Isso pode ser observado para as seis diferentes microrregiões estudadas (Tabela 3 ).

As probabilidades de ocorrência de seca, de acordo com SPI-1 e SPI-3, para as diferentes classes, foram semelhantes em todas as microrregiões, e variaram de 1 a $3 \%$ para eventos extremamente secos (Tabela 3 ). A semelhança entre essas escalas temporais também foi observada por Keskin \& Sorman (2010), que afirmaram que ambas as escalas poderiam ilustrar os efeitos da seca na agricultura.

Entre as microrregiões estudadas, a do sudoeste de Goiás apresentou maior ocorrência de eventos de seca extremos de curta duração. Para as escalas mensal e trimestral(SPI-1 e SPI-3), esta microrregião apresentou uma probabilidade de 2 e $3 \%$ para essas classes de evento, respectivamente (Tabela 3). Pelo SPI-12, a probabilidade de ocorrência de eventos extremamente secos nessa região foi nula. Isso talvez explique o fato de essa microrregião se destacar no cenário agrícola nacional.

A microrregião Meia-Ponte apresentou maior probabilidade de ocorrência (6\%) de eventos extremos de seca de maior persistência (SPI-12), seguida das microrregiões Entorno de Brasília e Porangatu (5\%), e Catalão e Rio Vermelho (3\%). Assim, com base na distribuição geográfica das seis microrregiões estudadas, pode-se inferir que as regiões sul e norte-nordeste do Estado de Goiás são mais propensas a eventos de seca de maior persistência.

A confrontação dos dados das séries temporais de produtividade ajustada do arroz de terras altas com o SPI, nas diferentes escalas, permitiu observar o desempenho dessas escalas em estimar a variação (diminuição e aumento) na produtividade ajustada da cultura (Figura 3). A diminuição da produtividade ajustada ocorreu em: 12 anos, na microrregião de Catalão; 13 anos, nas microrregiões Entorno de Brasília, Rio Vermelho e Meia-Ponte; e 14 anos, no sudoeste de Goiás e Porangatu. O número de eventos relativos à diminuição da produtividade ajustada, nas seis microrregiões utilizadas no presente trabalho, está próximos ao dos encontrados por Fernandes et al. (2010), que observaram diminuição em 13 anos, na série histórica de dados de produtividade de arroz de

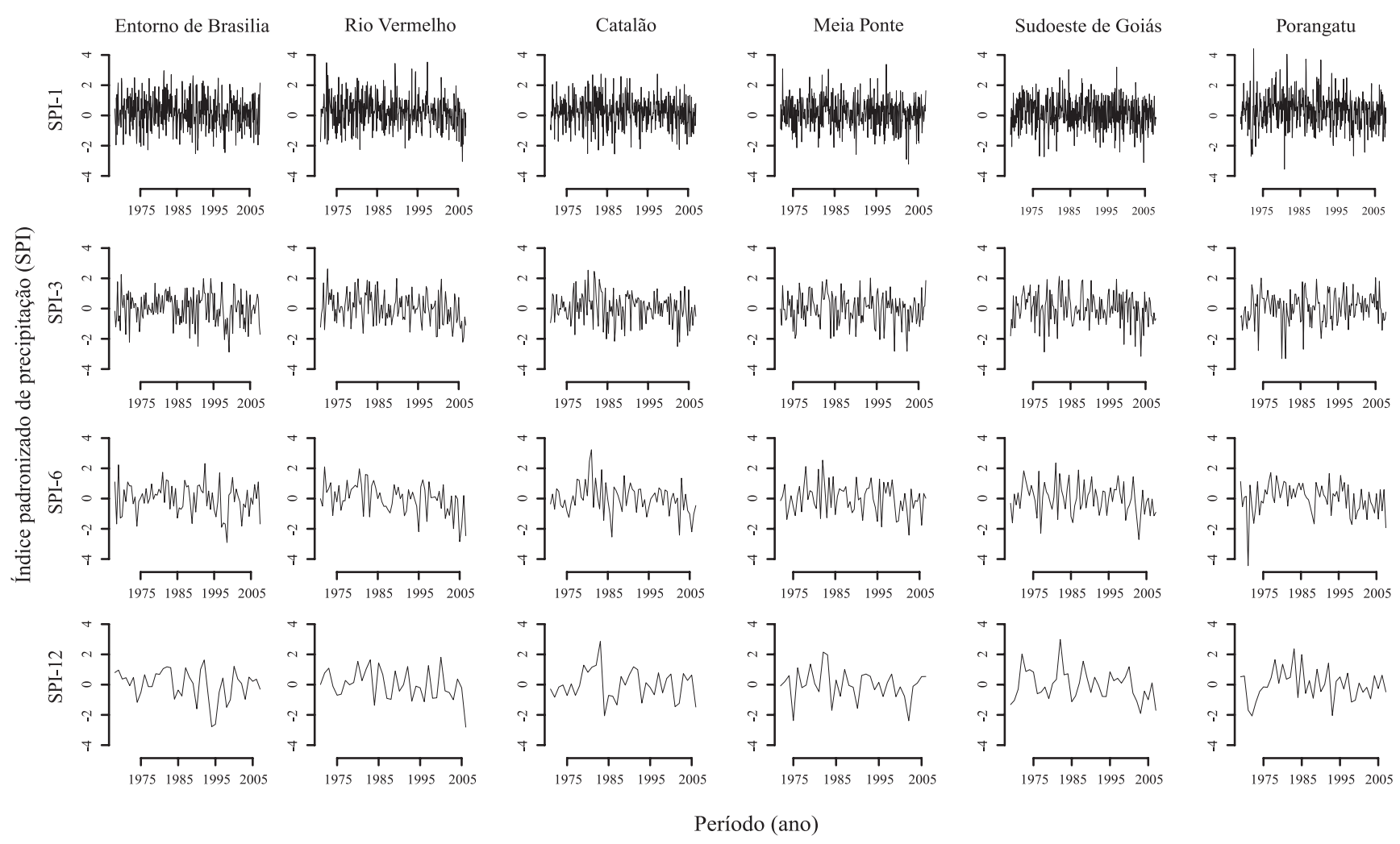

Figura 2. Séries temporais do índice padronizado de precipitação (SPI), em diferentes escalas temporais, nas seis microrregiões analisadas do Estado de Goiás. SPI-1, escala mensal; SPI-3, escala trimestral; SPI-6, escala semestral; e SPI-12, escala anual. 
terras altas, na microrregião de Goiânia, situada entre o Entorno de Brasília e Rio Vermelho, para o mesmo período de dados de produtividade utilizados no presente trabalho. O SPI acumulado que apresentou o melhor desempenho em estimar a variação na produtividade ajustada foi o SPI-12.ac, com índice de acerto igual

Tabela 3. Número e probabilidade de ocorrência de eventos secos ou úmidos, em seis microrregiões do Estado de Goiás, de acordo com o índice padronizado de precipitação (SPI) nas escalas mensal (SPI-1), trimestral (SPI-3), semestral (SPI-6) e anual (SPI-12).

\begin{tabular}{|c|c|c|c|c|c|c|c|c|}
\hline \multirow[t]{2}{*}{ Classe } & \multicolumn{4}{|c|}{ Número de ocorrências } & \multicolumn{4}{|c|}{ Probabilidade de ocorrência (\%) } \\
\hline & SPI-1 & SPI-3 & SPI-6 & SPI-12 & SPI-1 & SPI-3 & SPI-6 & SPI-12 \\
\hline & \multicolumn{8}{|c|}{ Entorno de Brasília } \\
\hline$\geq 2,00$ & 20 & 1 & 2 & 0 & 4 & 1 & 3 & 0 \\
\hline 1,00 a 1,99 & 77 & 26 & 8 & 7 & 16 & 16 & 10 & 18 \\
\hline 0,50 a 0,99 & 64 & 25 & 17 & 7 & 14 & 16 & 22 & 18 \\
\hline$-0,49$ a 0,49 & 199 & 63 & 29 & 14 & 43 & 39 & 37 & 35 \\
\hline$-0,99$ a $-0,50$ & 53 & 19 & 9 & 6 & 11 & 12 & 12 & 15 \\
\hline$-1,99$ a $-1,00$ & 48 & 22 & 12 & 4 & 10 & 14 & 15 & 10 \\
\hline \multirow[t]{2}{*}{$\leq-2,00$} & 7 & 4 & 1 & 2 & 1 & 3 & 1 & 5 \\
\hline & \multicolumn{8}{|c|}{ Rio Vermelho } \\
\hline$\geq 2,00$ & 18 & 1 & 1 & 0 & 4 & 1 & 1 & 0 \\
\hline 1,00 a 1,99 & 63 & 24 & 8 & 8 & 15 & 17 & 11 & 22 \\
\hline 0,50 a 0,99 & 57 & 19 & 15 & 3 & 13 & 13 & 21 & 8 \\
\hline$-0,49$ a 0,49 & 199 & 56 & 26 & 13 & 46 & 39 & 36 & 36 \\
\hline$-0,99$ a $-0,50$ & 47 & 18 & 10 & 7 & 11 & 13 & 14 & 19 \\
\hline$-1,99$ a $-1,00$ & 44 & 25 & 9 & 4 & 10 & 17 & 13 & 11 \\
\hline \multirow[t]{2}{*}{$\leq-2,00$} & 4 & 1 & 3 & 1 & 1 & 1 & 4 & 3 \\
\hline & \multicolumn{8}{|c|}{ Catalão } \\
\hline$\geq 2,00$ & 14 & 2 & 2 & 1 & 3 & 1 & 3 & 3 \\
\hline 1,00 a 1,99 & 65 & 17 & 9 & 4 & 15 & 12 & 13 & 11 \\
\hline 0,50 a 0,99 & 77 & 31 & 9 & 8 & 18 & 22 & 13 & 22 \\
\hline$-0,49$ a 0,49 & 197 & 57 & 31 & 10 & 46 & 40 & 43 & 28 \\
\hline$-0,99$ a $-0,50$ & 31 & 14 & 12 & 7 & 7 & 10 & 17 & 19 \\
\hline$-1,99$ a $-1,00$ & 41 & 19 & 6 & 5 & 9 & 13 & 8 & 14 \\
\hline \multirow[t]{2}{*}{$\leq-2,00$} & 7 & 4 & 3 & 1 & 2 & 3 & 4 & 3 \\
\hline & \multicolumn{8}{|c|}{ Meia-Ponte } \\
\hline$\geq 2,00$ & 9 & 1 & 2 & 1 & 2 & 1 & 3 & 3 \\
\hline 1,00 a 1,99 & 68 & 22 & 9 & 4 & 16 & 16 & 13 & 11 \\
\hline 0,50 a 0,99 & 79 & 25 & 11 & 7 & 19 & 18 & 16 & 20 \\
\hline$-0,49$ a 0,49 & 157 & 53 & 24 & 15 & 37 & 38 & 34 & 43 \\
\hline$-0,99$ a $-0,50$ & 60 & 16 & 12 & 4 & 14 & 11 & 17 & 11 \\
\hline$-1,99$ a $-1,00$ & 41 & 20 & 11 & 2 & 10 & 14 & 16 & 6 \\
\hline \multirow[t]{2}{*}{$\leq-2,00$} & 6 & 3 & 1 & 2 & 1 & 2 & 1 & 6 \\
\hline & \multicolumn{8}{|c|}{ Sudoeste de Goiás } \\
\hline$\geq 2,00$ & 9 & 1 & 1 & 2 & 2 & 1 & 1 & 5 \\
\hline 1,00 a 1,99 & 86 & 22 & 12 & 2 & 18 & 14 & 15 & 5 \\
\hline 0,50 a 0,99 & 75 & 28 & 11 & 7 & 16 & 18 & 14 & 18 \\
\hline$-0,49$ a 0,49 & 175 & 59 & 30 & 14 & 37 & 38 & 38 & 36 \\
\hline$-0,99$ a $-0,50$ & 65 & 22 & 9 & 7 & 14 & 14 & 12 & 18 \\
\hline$-1,99$ a $-1,00$ & 47 & 20 & 13 & 7 & 10 & 13 & 17 & 18 \\
\hline \multirow[t]{2}{*}{$\leq-2,00$} & 11 & 4 & 2 & 0 & 2 & 3 & 3 & 0 \\
\hline & \multicolumn{8}{|c|}{ Porangatu } \\
\hline$\geq 2,00$ & 26 & 2 & 0 & 1 & 6 & 1 & 0 & 3 \\
\hline 1,00 a 1,99 & 58 & 20 & 13 & 5 & 12 & 13 & 17 & 13 \\
\hline 0,50 a 0,99 & 126 & 27 & 14 & 6 & 27 & 17 & 18 & 15 \\
\hline$-0,49$ a 0,49 & 158 & 65 & 27 & 16 & 34 & 42 & 35 & 41 \\
\hline$-0,99$ a $-0,50$ & 52 & 24 & 12 & 5 & 11 & 15 & 15 & 13 \\
\hline$-1,99$ a $-1,00$ & 39 & 14 & 11 & 4 & 8 & 9 & 14 & 10 \\
\hline$\leq-2,00$ & 9 & 4 & 1 & 2 & 2 & 3 & 1 & 5 \\
\hline
\end{tabular}


ou maior que $50 \%$, para cinco das seis microrregiões estudadas (Porangatu, Meia Ponte, Catalão, Entorno de Brasília e Sudoeste de Goiás), seguido dos SPI-6.ac, SPI-3.ac e SPI-1.ac, com acertos iguais ou maiores que $50 \%$ para quatro microrregiões (Figura 4). O SPI-12. ac também foi o que apresentou o maior percentual de acerto, na estimativa da variação da produtividade ajustada, nas microrregiões de Catalão, Meia-Ponte e Porangatu. A provável razão de o SPI-12.ac ter apresentado esse melhor desempenho é o fato de esta escala temporal ser mais eficiente em detectar eventos extremos de maior persistência e, portanto, com maior potencial de impacto na produtividade agrícola. É importante salientar que os valores de produtividade ajustada provêem de uma estimativa média de produtividade referente à microrregião, o que, de certo modo, colabora para minimizar o efeito negativo de eventos não extremos.

A relação entre os desvios da produtividade ajustada e os valores obtidos pelo SPI.ac, para as quatro escalas temporais, ilustra os efeitos dos diferentes eventos de seca e umidade, obtidos pelo SPI.ac, na produtividade ajustada (Figura 5). Os valores obtidos pelo SPI-1. ac e SPI-3.ac, para eventos extremos $(-2,0 \leq \mathrm{SPI} \geq 2,0)$, não ilustraram ocorrência significativa de desvios negativos na produtividade ajustada (Figuras $5 \mathrm{~A}$ e B). Isso corrobora o que foi discutido anteriormente: as escalas mensais e trimestrais têm o melhor desempenho para eventos extremos de menor persistência, ou seja, de menor impacto negativo na produtividade ajustada das microrregiões. A escala semestral SPI-6. ac detectou a ocorrência de um maior número de eventos extremos, com capacidade de causar desvio negativo na produtividade (Figura $5 \mathrm{C}$ ). Assim, esta escala identifica melhor os efeitos prejudiciais de eventos extremos sobre a produtividade ajustada das microrregiões.

As linhas de tendência entre os índices de seca e o desvio da produtividade ajustada do arroz de terras altas, para as escalas temporais mensal, trimestral e semestral, são representadas por um polinômio de segunda ordem (Figuras 5 A, B e C). De acordo com Ash et al. (1992), este fato aproxima a natureza da relação entre água e produtividade, ou seja, tanto o
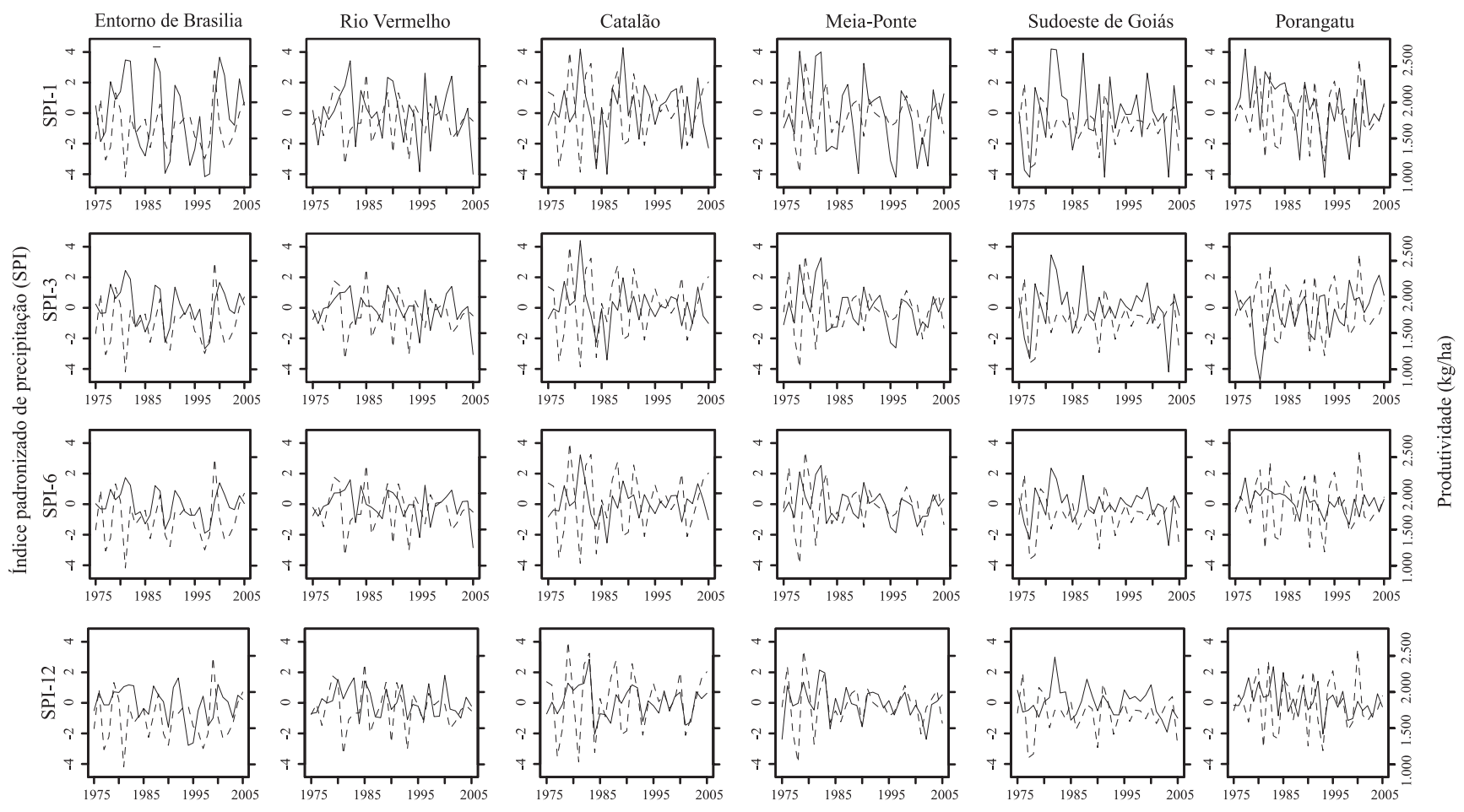

Período (ano)

- - - Produtividade $\left(\mathrm{kg} \mathrm{ha}^{-1}\right)-$ SPI

Figura 3. Comparação entre as séries temporais de produtividade de arroz de terras altas e o índice padronizado de precipitação (SPI) acumulado, para as diferentes escalas temporais e microrregiões. 


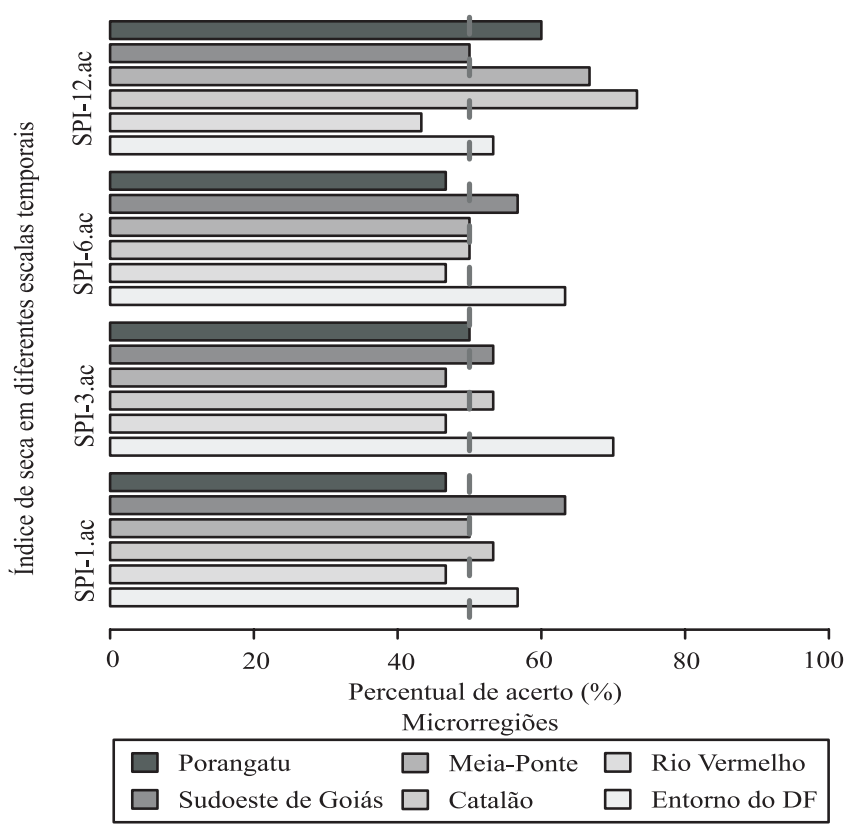

Figura 4. Percentual de acerto na variação da produtividade ajustada de arroz de terras altas, nas seis microrregiões estudadas do Estado de Goiás, de acordo com o uso de índices padronizados de precipitação (SPI) acumulados: SPI-1.ac, escala mensal; SPI-3.ac, escala trimestral; SPI-6. ac, escala semestral; e SPI-12.ac, escala anual.
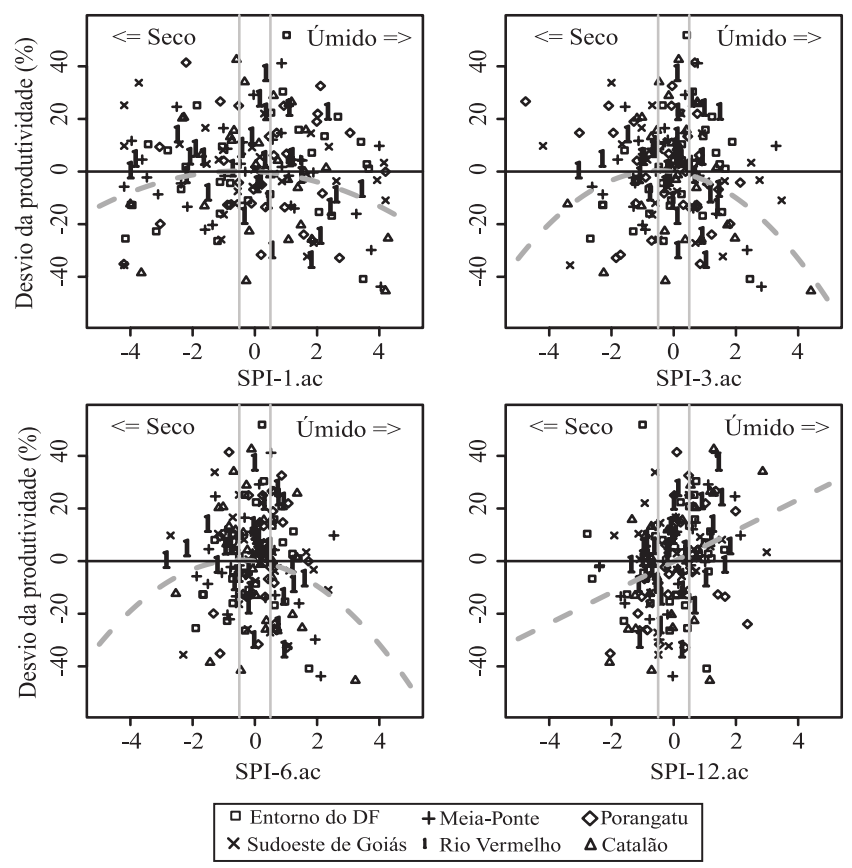

Figura 5. Relação entre o desvio da produtividade ajustada de arroz de terras altas, em relação ao valor médio, e os valores do SPI acumulados, com suas respectivas linhas de tendência (linha tracejada cinza): SPI-1.ac, escala mensal; SPI-3.ac, escala trimestral; SPI-6.ac, escala semestral; e SPI-12.ac, escala anual. excesso como a falta d'água são prejudiciais. Para a escala anual, os valores negativos de SPI-12.ac (abaixo de -0,49) concentraram basicamente desvios negativos na produtividade, enquanto valores positivos (acima de 0,49) concentraram, em sua maioria, desvios positivos. Diferentemente das outras escalas temporais, que apresentaram tendência quadrática entre índice de seca e desvio de produtividade ajustada, o SPI-12.ac apresentou tendência linear (Figura 5 D). Assim, pode-se inferir que, nessa escala temporal, os eventos de seca possuem maior impacto negativo na produtividade do que os eventos úmidos. Isso também ajuda a explicar porque os valores de SPI-12. ac possuem o melhor desempenho na identificação da variabilidade, na produtividade ajustada de arroz de terras altas, nas microrregiões do Estado de Goiás.

\section{Conclusões}

1. Quanto maior a escala temporal utilizada para obter os valores de SPI, mais persistentes são os eventos de seca identificados.

2. As regiões sul e norte-nordeste do Estado de Goiás são mais propensas a eventos de secas mais persistentes.

3. A escala temporal anual apresenta o melhor desempenho na estimativa da variação da produtividade ajustada de arroz de terras altas, nas microrregiões do Estado de Goiás.

\section{Referências}

ASH, G.H.B.; SHAYKEWICH, C.F.; RADDATZ, R.L. Moisture risk assessment for spring wheat on the Eastern prairies: a water-use simulation model. Climatological Bulletin, v.26, p.65-78, 1992.

BRASIL. Ministério do Meio Ambiente. Agência Nacional das Águas. Agência Nacional das Águas [home page]. Disponível em: <http://www.ana.gov.br>. Acesso em: 11 maio 2011.

BURKE, E.J.; BROWN, S.J.; CHRISTIDIS, N. Modeling the recent evolution of global drought and projections for the twenty-first century with the Hadley Centre climate model. Journal of Hydrometeorology, v.7, p.1113-1125, 2005.

CLEVELAND, W.S. Robust locally weighted regression and smoothing scatterplots. Journal of the American Statistical Association, v.74, p.829-836, 1979.

FATHABADI, A.; GHOLAMI, H.; SALAJEGUE, A.; AZANIVAND, H.; FATHABADI, A.; KHOSRAVI, H. Drought forecasting using neural network and stochastic models. Advances in Natural and Applied Sciences, v.3, p.137-146, 2009. 
FERNANDES, D.S.; HEINEMANN, A.B.; PAZ, R.L.F.; AMORIM, A. de O. Desempenho de índices quantitativos de seca na estimativa da produtividade de arroz de terras altas. Pesquisa Agropecuária Brasileira, v.45, p.771-779, 2010.

FREITAS, M.A. de S. Um sistema de suporte à decisão para o monitoramento de secas meteorológicas em regiões semi-áridas. Revista Tecnologia, p.84-95, 2005. Suplemento.

GARAGORRY, F.L.; REGO, A.M. AGROTEC: base relacional de dados estatísticos: estrutura de dados. Versão 1.0. Brasília: Embrapa-CPAC, 1997. 20p.

GIDDINGS, L.; SOTO, M.; RUTHERFORD, B.M.; MAAROUF, A. Standardized precipitation index zones for Mexico. Atmosfera, v.18, p.33-56, 2005.

GUTTMAN, N.B. Comparing the Palmer drought index and the standardized precipitation index. Journal of the American Water Resources Association, v.34, p.113-121, 1998.

HEIM, R.R. A review of twentieth-century drought indices used in the United States. Bulletin of the American Meteorological Society, v.83, p.1149-1163, 2002.

HEINEMANN, A.B.; SILVA, S.C. da; LOPES JUNIOR, S.; AMORIM, A. de O.; ANDRADE, C. de L.T. de; BASTOS, E.A.; PAZ, R.L. da. Características climáticas dos municípios de Santo Antônio de Goiás (GO), Porangatu (GO), Janaúba (MG), Sete Lagoas (MG), Parnaíba (PI) e Teresina (PI), Brasil. Santo Antônio de Goiás: Embrapa Arroz e Feijão, 2007. 36p. (Embrapa Arroz e Feijão. Documentos, 214).

HOLLINGER, S.E.; EHLER, E.J.; CARLSON, R.E. Midwestern United States corn and soybean yield response to changing El Niño-Southern Oscillation conditions during the growing season. In: HATFIELD, J.L.; VOLENEC, J.J.; DICK, W.A.; KRAL, D.M. (Ed.). Impacts of EI Niño and climate variability on agriculture. Madison: American Society of Agronomy, 2001. p.31-54. (ASA. Special publication, 63).

KELlER FILHO, T.; ASSAD, E.D.; LIMA, P.R.S. de R. Regiões pluviometricamente homogêneas no Brasil. Pesquisa Agropecuária Brasileira, v.40, p.311-322, 2005.

KESKIN, F.; SORMAN, U. Assesment of the drought pattern change in Çamlidere basin using SPI index. In: CONFERENCE ON WATER OBSERVATION AND INFORMATION SYSTEM FOR DECISION SUPPORT, 4, 2010, Ohrid. Proceedings. Ohrid: Geological Society of South Africa, 2010. p.1-8.
LIVADA, I.; ASSIMAKOPOULOS, V.D. Spatial and temporal analysis of drought in Greece using the standardized precipitation index (SPI). Theoretical and Applied Climatology, v.89, p.143-153, 2007.

LLOYD-HUGHES, B.; SAUNDERS, M.A. A drought climatology for Europe. International Journal of Climatology, v.22, p.1571-1592, 2002.

MCKEE, T.B.; DOESKEN, N.J.; KLEIST, J. The relationship of drought frequency and duration to the time scales. In: CONFERENCE ON APPLIED CLIMATOLOGY, 8, 1993, Anaheim. Proceedings. Boston: American Meteorological Society, 1993. p.179-184.

MICHAELIDES, S.; PASHIARDIS, S. Monitoring drought in Cyprus during the 2007-2008 hydrometeorological year by using the standardized precipitation index (SPI). European Water, v.23/24, p.123-131, 2008.

NIMER, E. Climatologia do Brasil. 2.ed. Rio de Janeiro: IBGE, 1989. 421p.

PINHEIRO, B. da S. Características morfofisiológicas da planta relacionadas à produtividade. In: SANTOS, A.B. dos; STONE, L.F.; VIEIRA, N.R. de A. (Ed.). A cultura do arroz no Brasil. 2.ed. rev. ampl. Santo Antônio de Goiás: Embrapa Arroz e Feijão, 2006. p.209-256.

POTOP, V.; TURKOTT, L.; KOZNAROVÁ, V.; MOZNÝ, M. Drought episodes in the Czech Republic and their potential effects in agriculture. Theoretical and Applied Climatology, v.99, p.373-388, 2010.

SALAS, J.D.; FU, C.; CANCELLIERE, A.; DUSTIN, D.; BODE, D.; PINEDA, A.; VINCENT, E. Characterizing the severity and risk of drought in the Poudre River, Colorado. Journal of Water Resources Planning and Management, v.131, p.383-393, 2005.

THOM, H.C.S. A note on the gamma distribution. Monthly Weather Review, v.86, p.117-122, 1958.

VICENTE-SERRANO, S.M.; BEGUERÍA, S.; LÓPEZ-MORENO, J. A multiscalar drought index sensitive to global warming: the standardized precipitation evapotranspiration index. Journal of Climate, v.23, p.1696-1718, 2010.

WU, H.; SVOBODA, M.D.; HAYES, M.J.; WILHITE, D.A.; WEN, F. Appropriate application of the standardized precipitation index in arid locations and dry seasons. International Journal of Climatology, v.27, p.65-79, 2005.

Recebido em 16 de novembro de 2010 e aprovado em 3 de março de 2011 